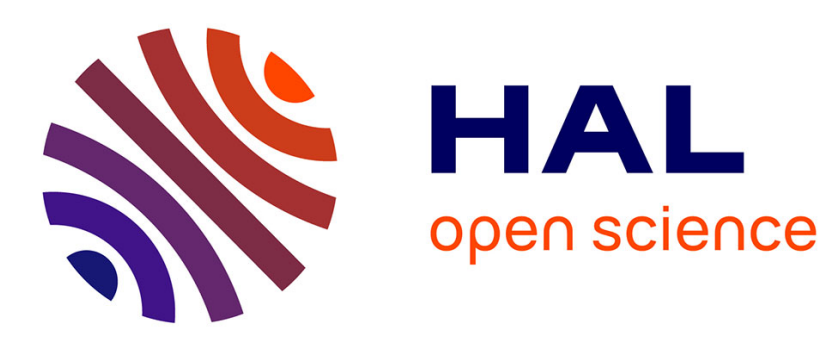

\title{
Nouveaux indices de variabilité au sein des traditions funéraires de l'ouest du Rubané occidental
}

\author{
Laura Waldvogel
}

\section{To cite this version:}

Laura Waldvogel. Nouveaux indices de variabilité au sein des traditions funéraires de l'ouest du Rubané occidental. Archimède: archéologie et histoire ancienne, 2021, Archimède. Archéologie et histoire ancienne, 8, pp.128-140. 10.47245/archimede.0008.ds2.04 . halshs-03281430

\section{HAL Id: halshs-03281430 \\ https://shs.hal.science/halshs-03281430}

Submitted on 8 Jul 2021

HAL is a multi-disciplinary open access archive for the deposit and dissemination of scientific research documents, whether they are published or not. The documents may come from teaching and research institutions in France or abroad, or from public or private research centers.
L'archive ouverte pluridisciplinaire HAL, est destinée au dépôt et à la diffusion de documents scientifiques de niveau recherche, publiés ou non, émanant des établissements d'enseignement et de recherche français ou étrangers, des laboratoires publics ou privés. 
1 DOSSIER THÉMATIQUE 1

SIUE DEUS SIUE DEA. DÉNOMINATIONS DIVINES DANS LES MONDES GREC ET SÉMITIQUE : UNE APPROCHE PAR LE GENRE

DOSSIER THÉMATIQUE 2

TRADITION ET TRANSMISSION DANS L'ANTIQUITÉ : RÉFLEXIONS INTERDISCIPLINAIRES

98 Claire CAMBerlein, Efstathia Dionysopoulou \& Thibault Foulon

Introduction. La tradition et sa transmission : positionnements théoriques

105 Mélissa Leuzy

Consigner ou fabriquer la légende d'Alexandre le Grand ? Arrien face à la tradition dans l'Anabase

117 Héloïse SMETS

Un autre regard sur l'archaïsme dans les sépultures privées de l'Égypte pharaonique

128 Laura WALDVOGEL

Nouveaux indices de variabilité au sein des traditions funéraires de l'ouest du Rubané occidental

141 Grégoire BLANC

Praxis citationnelle et co-construction du discours scientifique dans les Naturales Quaestiones de Sénèque (livres II \& III)

150 Anthony GLAISE

Polémiques, traditions et identités : réflexions autour des Discours contre les juifs et les judaïsants de Jean Chrysostome

159 ACTUALITÉ DE LA RECHERCHE

QUOI DE NEUF À L'OUEST DE STRASBOURG ? KOENIGSHOFFEN : ÉTAT DES LIEUX ET DÉCOUVERTES RÉCENTES

195 VARIA 


\title{
NOUVEAUX INDICES DE VARIABILITÉ AU SEIN DES TRADITIONS FUNÉRAIRES DE L'OUEST DU RUBANÉ OCCIDENTAL
}

\author{
Laura WALDVOGEL \\ Doctorante en archéologie préhistorique \\ Université de Strasbourg \\ UMR 7044 Archimède
}

laura.waldvogel@etu.unistra.fr

\section{RÉSUMÉ}

En 1995, Ch. Jeunesse distingue deux traditions funéraires à l'ouest du Rubané occidental, scindant la plaine d'Alsace en deux zones : la Haute-Alsace d'une part (tradition I) et la Basse-Alsace d'autre part (tradition II). Notre étude du degré de flexion des membres inférieurs des défunts de ces deux régions, conduite dans le cadre d'une thèse de doctorat, permet non seulement d'établir une nouvelle distinction entre les traditions I et II, mais également entre certains groupes appartenant à la même tradition. Ces résultats, cumulés à d'autres éléments ayant trait tant à la structure des fosses sépulcrales qu'à la composition du mobilier funéraire, suggèrent l'existence d'une variabilité géographique. Ils permettent

\section{MotS-CLÉs}

Néolithique ancien danubien, pratiques funéraires, Alsace,

sépultures,

Rubané occidental. ainsi d'identifier au moins deux sous-groupes au sein de chacun de ces deux ensembles à partir du Rubané récent.
In 1995, Ch. Jeunesse identified two funerary traditions within the western part of western LBK, dividing the Alsace plain into two areas: Southern Alsace (tradition I) and Northern Alsace (tradition II). The study of the degree of flexion of the lower limbs of the deceased in these two regions, conducted within the framework of a PhD thesis, establishes a new distinction between tradition I and II, as well as between several groups belonging to the same tradition. These results, combined with other elements linked to the structure of the graves and the nature of funeral goods, suggest the existence of geographical variability among the same tradition. They make it possible to outline at least two sub-groups within both tradition I and II from the LBK recent stage.
KEYWORDS

Danubian Neolithic, funerary practices, Alsace, burials, Western LBK. 
Cœur de la discipline ethnologique, la notion de « tradition » a pourtant rarement donné lieu à des tentatives de caractérisation de son objet : G. Lenclud lui confère ainsi le statut de «mot-outil » [1], en cela qu'il ne permet qu'une identification évasive du concept - et non de l'objet - auquel il réfère. La majorité des critiques faites à son encontre semble néanmoins s'accorder sur un point : la «tradition » consisterait au moins en la transmission de génération en génération, et ce dans un but conservatoire, d'une « chose, un énoncé ou une manière de faire (ou de dire) 》[2] ayant une valeur culturelle intrinsèque. Elle est «un passé [qui] persiste dans le présent où elle est transmise et demeure agissante et acceptée par ceux qui la reçoivent et qui, à leur tour, au fil des générations la transmettent » [3] par voie essentiellement non écrite (la parole) ou par l'exemple [4]. Elle est, en ce sens, une « invention » du présent constituant « un mode de légitimation de l'identité et de l'appartenance collective qui s'inscrit dans une dialectique de l'en-groupe et du hors-groupe »[5]. Transposés à I'archéologie funéraire, ces premiers éléments de définition suggèrent un rapprochement avec la notion de «pratique » (au sens de « comportement habituel »[6]), bien que la «tradition » s'en distingue par son aspect profondément culturel. Elle se caractérise en effet par un « discours » hautement symbolique consistant entre autres à déposer dans les tombes, et ce de manière récurrente, des objets signifiants sur une période assez longue pour couvrir plusieurs générations. Des communautés partageant une même culture, au

[1] Bouju 1995, p. 95.

[2] Ibid.

[3] PouilLon 1991, p. 710.

[4] LENCLUD 1987, p. 110-113.

[5] Bouju 1995, p. 107.

[6] Boulestin 2016, p. 376. On préférera ainsi le terme de « pratique » à celui de «norme », souvent mésusé et empêchant par nature la mise en évidence d'une variabilité funéraire.

[7] La notion de culture, telle qu'envisagée par les anthropologues, a donné lieu à des définitions substantielles depuis celle proposée par E. B. Tylor dans Primitive Culture (1871). Nous nous arrêterons ici sur celle de C. Lévi-Strauss, qui la perçoit comme un ensemble de systèmes symboliques dont l'objectif est « d'exprimer certains aspects de la réalité physique et de la réalité sociale, et, plus encore, les relations que ces deux types de réalité entretiennent entre eux, et que les systèmes sens anthropologique du terme [7], peuvent néanmoins avoir recours à des traditions différentes [8] discernables à travers une variabilité diverse - toute permanence entraînant des variations [9] - qui aura pour conséquence d'altérer le « discours », sans toutefois modifier son essence. Tel est le cas du Rubané, culture archéologique [10] du Néolithique ancien dont la zone nucléaire se situe dans la région du Danube. L'étude de ces pratiques funéraires, entreprise par $\mathrm{Ch}$. Jeunesse au milieu des années 1990 [11], a permis d'identifier deux traditions [12] particulièrement visibles dans la partie ouest du Rubané occidental [13], parfois dès le Rubané ancien [14] : la tradition I, qui recouvre I'aire du Rubané du sud-ouest (RSO) et la tradition II, englobant les groupes du Rubané du nordouest (RNO), du Rubané du Neckar (RN) et du Rubané de Basse-Alsace (RBA). La plaine d'Alsace se trouve ainsi scindée en deux zones correspondant à celles des deux groupes céramiques identifiés dans la région (fig. 1). Jusqu'alors, les dissemblances fondamentales entre ces entités reposaient principalement sur la composition du mobilier funéraire. Notre analyse, qui s'appuie sur un corpus actualisé, s'est de facto concentrée sur la position de dépôt du défunt, cet « élément central autour duquel se sont ordonnés les gestes mortuaires »[15]. Ce paramètre constitue en effet, avec l'orientation, le cœur de la pratique funéraire [16] et est étroitement lié à l'identité collective d'un groupe. Il est l'aboutissement d'une série de gestes codifiés formant ensemble une véritable «grammaire » porteuse de sens pour la population « inhumante ».

symboliques eux-mêmes entretiennent les uns avec les autres » (introduction de C. Lévi-Strauss à Mauss 1950).

[8] LENCLUD 1987, p. 119-122.

[9] LENCLUD 1987, p. 113-116.

[10] Elle est conçue comme un faciès spatio-temporel identifiable par le biais de la culture matérielle.

[11] JEUNESSE 1995a.

[12] Celles-ci résultent probablement de l'emprunt de deux axes de colonisation différents depuis le Danube (JEUNESSE 1995a, p. 138-140; Jeunesse 1996, p. 253).

[13] Le Rubané occidental s'oppose au Rubané oriental, ou «Rubané de I'Alföld », par son style céramique distinct : le Rubané occidental étant issu de la culture de Starčevo, et le Rubané oriental de celle de Köros (Jeunesse 1997). [14] C'est par exemple le cas en Allemagne centrale et en Basse-Bavière (Jeunesse 1996, p. 256).

[15] Duday et al. 1990, p. 30.

[16] JeUnESSE 1997, p. 106. 


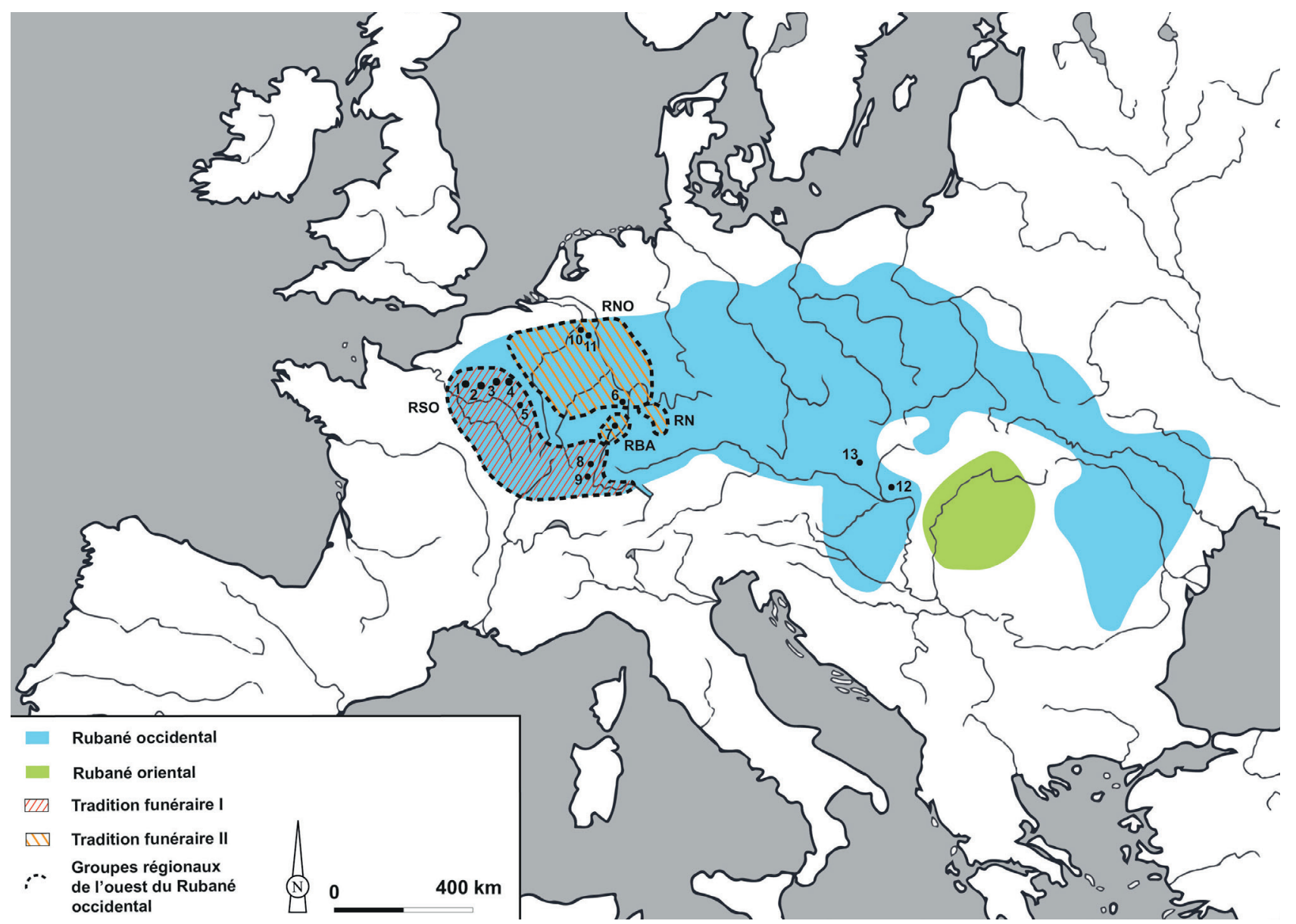

Fig. 1 : situation des traditions funéraires I et II de l'ouest du Rubané occidental. Principaux sites mentionnés dans le texte : 1, Bucy-le-Long «La Fosselle »; 2, Maizy-sur-Aisne « Les Grands Aisements »; 3, Berry-au-Bac « Le Vieux Tordoir » ; 4, Menneville « Derrière le Village » ; 5, Écriennes « La Folie » ; 6, Schwetzingen ; 7, Vendenheim « Le Haut du Coteau »; 8, Ensisheim « Les Octrois »; 9, Mulhouse-Est ; 10, Elsloo ; 11, Niedermerz ; 12, Nitra ; 13, Vedrovice «Široká u lesa » ; Groupes régionaux : 1, RBA : Rubané de Basse-Alsace ; 2, RN : Rubané du Neckar ; 3, RNO : Rubané du nord-ouest ; 4, RSO : Rubané du sud-ouest.

DAO : Laura Waldvogel, modifié d'après JEUNESSE 2003, fig. 1.

\section{LES TRADITIONS FUNÉRAIRES DU RUBANÉ OCCIDENTAL}

\section{TRADITION I (RUBANÉ DU SUD-OUEST)}

Les sépultures relevant de la tradition I sont identifiables dès le Rubané moyen [17] et restent globalement fidèles à la pratique de la zone nucléaire du Rubané (région du Danube), bien qu'elles présentent des particularités géographiques [18]. Les défunts dont le contexte a pu être déterminé se trouvent soit dans des nécropoles (Haute-Alsace), soit dans les villages ou à proximité de ceux-ci (Haute-Alsace et Bassin parisien [19]). Ils reposent généralement au sein de fosses comportant des traces d'ocre saupoudrée et sont majoritairement fléchis sur le côté gauche, tête à l'est [20]. Le trait le plus distinctif de cette tradition réside dans la teneur du mobilier funéraire, qui se caractérise par une prévalence des éléments de parure en coquillage marin. Ces derniers se divisent en deux groupes - les parures « internationales », décelables
[17] JeUnesse 1995a, p. 129. Lors de la rédaction de cet article, I'unique sépulture de Haute-Alsace attribuée au Rubané ancien à partir du mobilier céramique était la tombe 1 de Wittenheim-Schoenensteinbach « Leimbuckel », découverte en 1930. Nos connaissances de l'étape ancienne de cette région seront cependant amenées à évoluer suite à la publication des résultats de la fouille de la nécropole d'Ensisheim « Reguisheimer Feld » (fouille Archéologie Alsace/INRAP, 2019).
[18] Voir infra.

[19] Bonnabel et al. 2003 ; Constantin et al. 2003

[20] À l'exception de 9 individus de Haute-Alsace déposés sur un axe allant de I'OSO à I'ONO : Brunstatt « Land Dudely Acker » (sépulture 1), Colmar « Route de Rouffach » (st. 65), Ensisheim « Les Octrois » (sépulture 14), Mulhouse-Est (sépulture 20), Wettolsheim « Aussere Kreuzfeld/Pariser Breite » (sépultures I, II, III et IV) et Wettolsheim « Koenigsbreite » (sépulture 2). 
sur l'ensemble de la zone d'extension du Rubané, - et les parures « occidentales », caractéristiques du RSO, dont la composition connaît des évolutions entre le Rubané moyen et le Rubané récent [21]. Au Rubané moyen, le premier groupe comprend ainsi des bracelets en spondyle, des spondyles fendus et des perles tubulaires (ex. nécropole de Mulhouse-Est [22]), tandis que la valve de spondyle perforée et les perles en spondyle sont privilégiées au cours de l'étape suivante. Quant aux parures « occidentales », elles incluent au Rubané moyen des bagues en os, des perles en Dentalium et de petites perles sur test de spondyle aux formes variables (triangulaires, discoïdes, trapézoïdales, etc.). L'étape récente voit la perduration de la perle discoïde sur test de spondyle et l'apparition de perles sur petits coquillages marins contemporains, tels que les Trivia monacha, les Nucella lapillus et les Colombella rustica [23].

\section{TRADITION II (RUBANÉ DU NORD-OUEST, RUBANÉ DU NECKAR, RUBANÉ DE BASSE- ALSACE)}

Contrairement au RSO, les tombes de la tradition II ne comportent que rarement des éléments de parures « internationales » ou « occidentales». Bien que leur absence soit imputable à l'acidité du sol dans les cas des nécropoles d'Elsloo [24] (66 inhumations et 47 crémations) et de Niedermerz [25] (102 inhumations et 11 crémations), leur présence anecdotique à Schwetzingen (203 inhumations, 15 crémations et 15 tombes sans restes humains) corrobore les résultats obtenus en Basse-Alsace [26]. Dans cette zone, les seules exceptions sont la tombe 2 d'Osthoffen-Breuschwickersheim [27] (7 perles tubulaires sur test de spondyle et 1 valve de spondyle biforée), les tombes 6 et 7 de Quatzenheim « Auf dem Spitzenpfad » [28] (13 et au moins 14 Nucella lapillus), ainsi que les tombes $S$ et 26 de Souffelweyersheim « Tuilerie Reiss und Bund » [29] (1 et 4 Nucella lapillus). L'analyse des parures de Basse-Alsace montre en effet une utilisation prédominante de petits gastéropodes terrestres ou de moules d'eau douce (Zebrina detrita,

[21] JEUNESSE 1995a, p. 127-129.

[22] Schweitzer \& Schweitzer 1977 ; Jeunesse et al. 2015.

[23] Ibid.

[24] MODDERMAN 1970.

[25] DOHRN-IHMIg 1983.

[26] La nécropole de Schwetzingen a livré 6 éléments en spondyle localisés dans les sépultures 48 ( 1 bracelet et 1 valve biforée), 99 ( 1 perle tubulaire), 151 ( 1 perle tubulaire) et 170 ( 2 perles tubulaires). À cela s'ajoute une chaîne composée de 19 coquilles de Nucella lapillus découverte dans la sépulture 98 (voir GERLING 2012).
Unio, Margaritifera), qui composent 10 des 15 parures en coquillage recensées dans la région. À l'inverse, ils ont uniquement été relevés sur 11 des 33 parures découvertes en Haute-Alsace et, dans 9 de ces cas, ils font partie d'un ensemble composite intégrant des coquillages marins. Cette rareté des parures « internationales » et « occidentales », visible à partir du Rubané récent dans les groupes du RNO, du RN et du RBA, doit probablement être consécutive d'une insertion moins importante dans les réseaux d'approvisionnement les reliant au Danube et à la façade atlantique (via les populations mésolithiques) [30]. Outre la parure, la tradition II se caractérise par la fréquence des outillages lithiques taillés et polis, de la céramique, de l'ocre en fragments ainsi que du matériel de mouture. L'étape récente/finale se distingue par ailleurs par une variabilité dans I'orientation et la position des défunts : on retrouve ainsi un taux important d'individus à orientation « antipodique » [31] (fig. 2) ou reposant sur le dos, les deux membres inférieurs strictement en extension. Cette position, absente de la tradition I et en rupture avec la pratique rubanée, est particulièrement perceptible en BasseAlsace (ex. Entzheim «sud-est de la gare » [32], Ingenheim «Bannenberg »[33], Quatzenheim «Auf dem Spitzenpfad » [34], Souffelweyersheim «Tuilerie Reiss und Bund » [35] ou encore Vendenheim «Le Haut du Coteau »[36]).

\section{$L A \ll G R A M M A I R E » D E S$ INDIVIDUS FLÉCHIS}

\section{MÉTHODOLOGIE}

L'étude de la position des défunts de l'ouest du Rubané occidental a jusqu'à présent mis en exergue une distinction entre sujets « fléchis sur le côté » et « allongés sur le dos, membres inférieurs en extension », sans se pencher sur les différents paramètres présentés par cette première catégorie. Nous nous sommes inspirés de l'approche systémique afin de dégager d'éventuelles règles organisationnelles régissant la position des individus déposés fléchis dans une

[27] FORRER 1916 ; TABORIN 1974.

[28] Stieber 1947 ; Stieber 1955 ; Jeunesse 2005.

[29] ULRICH 1939 ; ULRICH 1953.

[30] JeUNESSE 1995a, p. 130-131.

[31] Il s'agit d'individus déposés sur un axe inverse à la pratique rubanée, c'est-à-dire tête à l'ouest.

[32] FORRER 1912.

[33] Lefranc et al. 2014.

[34] Stieber 1947 ; Stieber 1955 ; JeUnesse 2005.

[35] ULRICH 1939 ; ULRICH 1953.

[36] JeUNESSE 2002. 
Fig. 2 : orientation (tête-pieds) des individus de la nécropole de Vendenheim

« Le Haut du Coteau » (Rubané récent/final).

DAO : Laura Waldvogel, modifié d'après JeUnesse 2002, fig. 10.

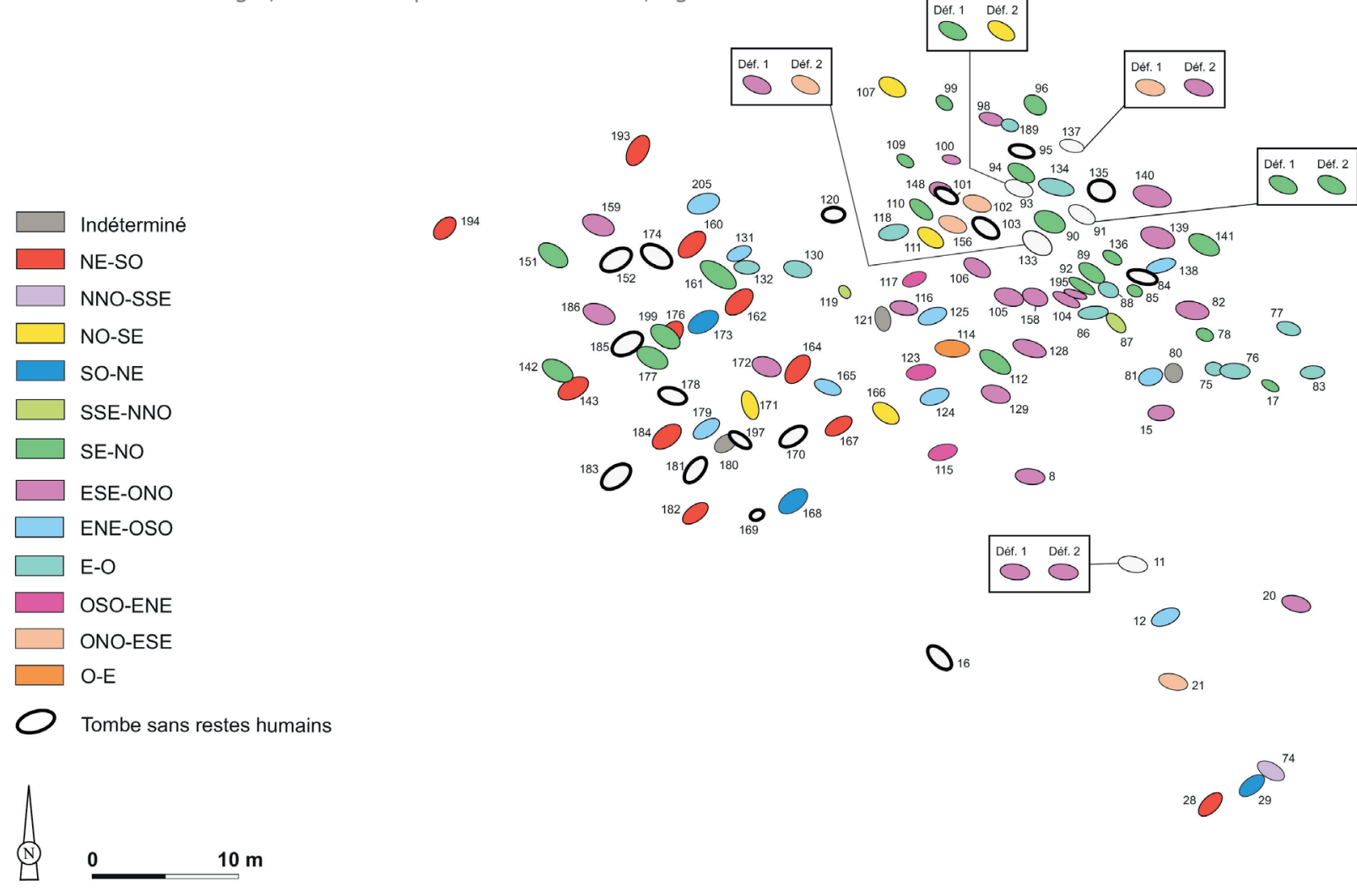

sépulture [37] et relevant des traditions I et II de la plaine d'Alsace. Notre démarche a consisté à analyser le degré de flexion du genou des défunts dans la perspective d'identifier de possibles dissemblances dans leur « grammaire ». Les membres inférieurs se prêtent en effet relativement bien à un tel exercice : leurs mouvements étant considérablement réduits en cas de dépôt en espace colmaté - un type d'espace qui constitue la majorité de notre corpus. Les données recueillies ont par ailleurs montré qu'une partie des sujets dont les membres inférieurs sont fléchis reposaient aussi bien sur le côté que sur le dos au moment de leur découverte. Ce constat reste le même si I'on prend en compte les déplacements osseux consécutifs à la décomposition : une partie de ces individus peut effectivement être inclue dans la catégorie des « fléchis sur le côté », mais quelques-uns semblent tout de même avoir initialement été mis en terre sur le dos, les membres inférieurs fléchis. Cette particularité

[37] Pour la notion de « sépulture », voir LECLERC 1990 ainsi que DudaY \& Boulestin 2005. Les individus datés du Rubané découverts en contexte d'habitat et déposés en position «conventionnelle » (fléchis sur le côté) ont été inclus dans notre étude. Ceux reposant en position «non conventionnelle » en sont, quant à eux, exclus.

[38] Nous avons identifié au moins 8 défunts déposés ainsi (sépultures 12,16, 48, 65, 117A, 157, 169 et 205). est perceptible à Schwetzingen [38], tout comme en Haute-Alsace (fig. 3 ) et, de manière beaucoup plus discrète à Nitra (Slovaquie) [39] et Vedrovice « Široká u lesa » (République Tchèque) [40]. Enfin, les tombes de la plaine d'Alsace se caractérisent par une documentation de qualité relativement hétérogène : il nous a uniquement été possible de procéder à un examen des découvertes les mieux renseignées. Le degré de flexion du genou a été déterminé suivant la convention en usage en anatomie humaine. Ainsi, une extension correspond à $0^{\circ}$, tandis qu'une flexion atteint une amplitude maximale de $160^{\circ}$ et se caractérise par la mise en contact du talon avec la fesse [41]. La mesure de ces angles a été effectuée au rapporteur pour les relevés de terrain et photographies en vue zénithale, et via un examen visuel pour les documents moins précis mais non moins fiables, tels que les croquis des défunts ou les photographies en vue non zénithale. Les plans de situation des tombes issues de certaines

[39] L'examen des photographies prises sur le terrain indique qu'au moins 3 défunts présentaient cette position (sépultures 14, 56 et 65). Voir Pavuk 1972.

[40] L'ensemble des sujets semble avoir été initialement inhumé sur le côté.

[41] KAPANDji 2018, p. 72. Une flexion totale, soit $180^{\circ}$, est quant à elle physiquement impossible, puisqu'elle est limitée par les masses musculaires. 


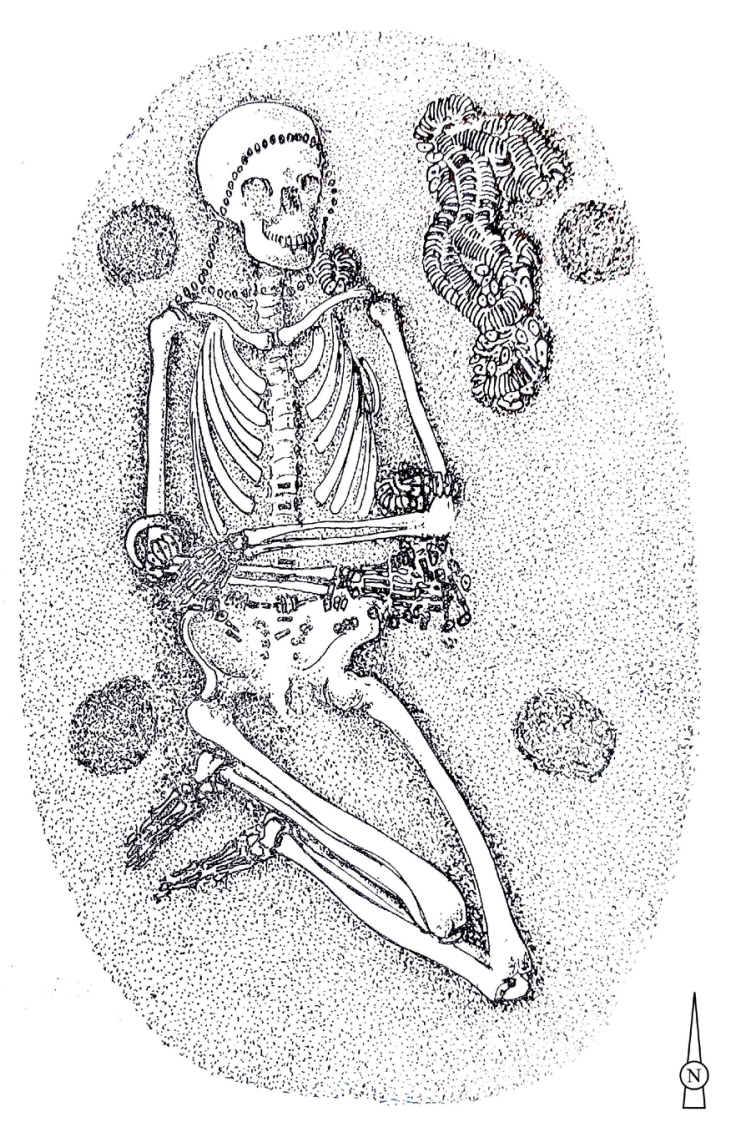

Fig. 3 : Défunt de la tombe 14 de la nécropole de Mulhouse-Est (Haute-Alsace), déposé sur le dos, les membres inférieurs hyperfléchis et rabattus vers la gauche. Dessin : Laura Waldvogel, modifié d'après Schweitzer \& Schweitzer 1977, pl. 14.

fouilles anciennes, sur lesquels figure parfois la position des individus, ont été écartés en raison de différences avec la position rapportée par les auteurs : tel est par exemple le cas des premières tombes découvertes à Souffelweyersheim « Tuilerie Reiss und Bund » [42]. Une fois ces données recueillies, nous avons calculé la moyenne du degré de flexion des deux membres inférieurs de chaque défunt [43] et l'avons reportée sur des graphiques divisés par étape chronologique et par aire géographique (Basse et Haute-Alsace).

[42] Voir notamment FORRER \& JÄNGER 1918

[43] Les individus dont seule la position de l'un des membres inférieurs est connue ont été écartés de notre analyse.

[44] Ce nombre exclut les inhumations non publiées ainsi que celles dont I'attribution au Rubané est incertaine et/ ou repose sur des éléments ténus.

[45] THEVENIN 1978.

[46] LeFrANc et al. 2004.

[47] LEFRANC 2012.

[48] JEUNESSE 1988.

[49] WolfF et al. 2000.

[50] Vergnaud et al. 2016.

\section{LE CORPUS}

Le nombre de sépultures rubanées mises au jour dans la plaine d'Alsace s'élevait à 348 lors de la rédaction du présent article [44] : parmi elles, 104 se trouvent en Haute-Alsace et 243 en Basse-Alsace. Il s'agit de :

- Tombes en contexte indéterminé (ex. Brunstatt « Lang Dudely Acker » [45]) ;

- Tombes disséminées dans l'emprise de villages (ex. Bischoffsheim «AFUA du stade » [46], Entzheim «Terres de la Chapelle - ZAC » [47] et Wettolsheim «Ricoh» [48]);

- Groupes de tombes en contexte indéterminé (ex. Geispitzen « Stuecke » [49] (5 sépultures) et Morschwiller-le-Bas « Ungeheuer Hoelzle » [50] (5 sépultures)). L'absence de groupes de tombes associés à un habitat dans la plaine d'Alsace, à l'image des découvertes faites dans le Bassin parisien, tend toutefois à suggérer leur appartenance à une nécropole ;

- Nécropoles situées à l'écart de villages (ex. Vendenheim « Le Haut du Coteau » (116 sépultures), Souffelweyersheim « Tuilerie Reiss und Bund » (52 sépultures), Mulhouse-Est (27 sépultures) ou encore Quatzenheim « Auf dem Spitzenpfad »(17 sépultures)).

Plusieurs d'entre elles n'ont pas pu être étudiées faute de photographies, de relevés ou de croquis des défunts. Notre étude a ainsi porté sur un total de 117 individus : 58 proviennent de 11 sites de Basse-Alsace et 59 de 8 sites de Haute-Alsace (fig. 4). Ils relèvent d'une étape indéterminée du Rubané, du Rubané moyen, du Rubané moyen ou récent, du Rubané récent ainsi que du Rubané récent ou final (fig. 5). Les étapes précédant le Rubané récent se caractérisent ainsi par un déséquilibre notable entre les deux régions : c'est particulièrement le cas du Rubané moyen, qui n'est pas attesté en Basse-Alsace, mais est bien représenté en Haute-Alsace.

\section{UNE « GRAMMAIRE » NON CONVENTION- NELLE PERCEPTIBLE EN BASSE-ALSACE DĖS LE RUBANÉ RÉCENT}

Les disparités entre Haute et Basse-Alsace permettent de comparer les données de ces deux zones uniquement à partir du Rubané récent. Elles n'empêchent toutefois pas d'observer les gestes du Rubané moyen en Haute-Alsace, où les membres inférieurs des individus sont systématiquement fléchis selon des angles n'allant jamais en deçà des $117^{\circ}$ (fig. 6). Ce constat est quasiment similaire au Rubané récent et final, puisque ces angles sont inférieurs à $120^{\circ}$ dans quatre cas seulement, sans toutefois passer sous la barre des $90^{\circ}$ (fig. 7). La mise en évidence de degrés de flexion allant 


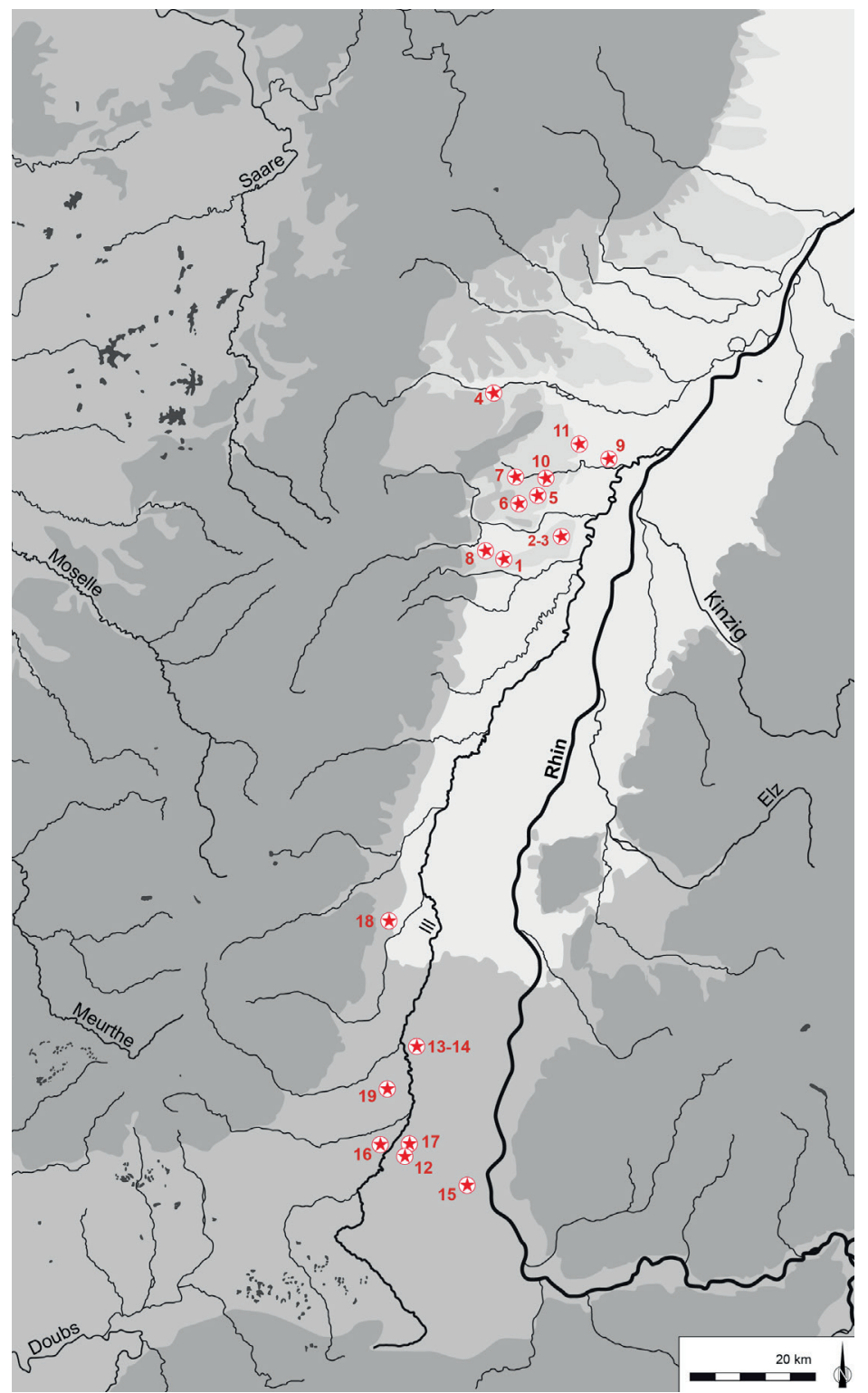

Fig. 4 : Localisation des sites du corpus.

1, Bischoffsheim «AFUA du stade »;

2, Entzheim « Sud-est de la gare »;

3, Entzheim « Terres de la Chapelle - ZAC » ;

4, Ingenheim « Bannenberg »;

5, Oberschaeffolsheim « Zwischen

Oberschaeffolsheim und Ittenheim »;

6, Osthoffen-Breuschwickersheim ;

7, Quatzenheim « Auf dem Spitzenpfad » :

8, Rosheim « ZAC du Rosenmeer -

Extension Baruch »;

9, Souffelweyersheim « Tuilerie

Reiss und Bund »;

10, Stutzenheim « Über dem Stutzenweg » ;

11, Vendenheim « Le Haut du Coteau »;

12, Brunstatt « Lang Dudely Acker »;

13, Ensisheim « Les Octrois » ;

14, Ensisheim « Ratfeld »;

15, Geispitzen « Stuecke »;

16, Morschwiller-le-Bas « Ungeheuer Hoelzle » ;

17, Mulhouse-Est ;

18, Wettolsheim « Ricoh » ;

19, Wittenheim « Le Moulin ».

DAO : Laura Waldvogel (fond

de carte: A. Denaire).

\begin{tabular}{|l|c|c|}
\cline { 2 - 3 } \multicolumn{1}{c|}{} & Basse Alsace & Haute Alsace \\
\hline Rubané indéterminé & 4 & 1 \\
\hline Rubané moyen & 0 & 16 \\
\hline Rubané moyen ou récent & 0 & 4 \\
\hline Rubané récent & 26 & 3 \\
\hline Rubané récent ou final & 28 & 35 \\
\hline Total & $\mathbf{5 8}$ & $\mathbf{5 9}$ \\
\hline
\end{tabular}

Fig. 5 : Nombre d'individus étudiés par étape chronologique en Basse et Haute-Alsace. DAO : Laura Waldvogel.

HAUTE ALSACE

Rubané moyen

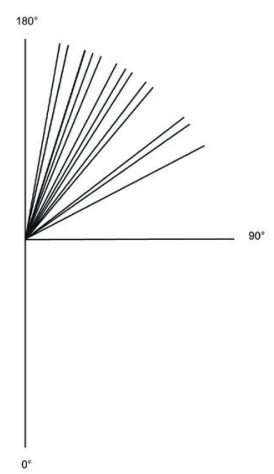

Fig. 6 : degrés de flexion des membres inférieurs des défunts de Haute-Alsace au Rubané moyen. DAO : Laura Waldvogel.
BASSE ALSACE

Rubané récent et final

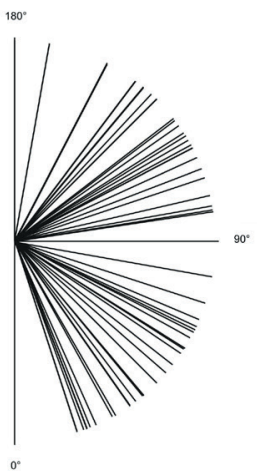

HAUTE ALSACE

Rubané récent et final

${ }^{180}$

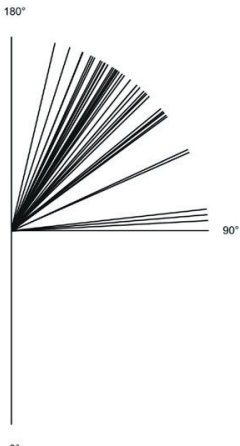

Fig. 7 : degrés de flexion des membres inférieurs des défunts de Basse et HauteAlsace au Rubané récent et final. DAO : Laura Waldvogel. 
au-delà de l'amplitude maximale du membre inférieur, soit $160^{\circ}$, suggère de fait le recours à un élément de type enveloppe très serrée ou liens ayant pour effet de prévenir les déplacements osseux hors du volume du corps lors de la décomposition des chairs. À l'inverse, les résultats de la Basse-Alsace mettent en lumière des fléchissements selon des degrés tout à fait aléatoires, compris entre environ $18^{\circ}$ et $170^{\circ}$ (fig. 7) : un phénomène qui semble être valable aussi bien pour les nécropoles que pour les tombes en habitat, comme le montre par exemple l'individu de la tombe 2005 d'Entzheim «Les Terres de la Chapelle - ZAC », dont la moyenne de flexion des membres inférieurs est de $56^{\circ}$. Ces dissemblances dans la « grammaire » des sujets de Basse et de Haute-Alsace se voient d'ailleurs confirmées par l'ajout des données des sujets datés d'une étape indéterminée du Rubané et du Rubané moyen ou récent (fig. 8).

L'application d'une méthodologie identique à Nitra (Rubané récent régional, correspondant au Rubané ancien et moyen de l'ouest du Rubané occidental) a permis d'étudier 32 défunts dont le degré de flexion minimal est de $127,5^{\circ}$ (fig. 9). Un résultat similaire a été mis en évidence pour la nécropole de Vedrovice « Široká u lesa » (Rubané le plus ancien/Rubané ancien), où l'écrasante majorité des 52 individus examinés présente des angles supérieurs à $120^{\circ}$ (fig. 9). L'étude de ces deux ensembles funéraires, localisés dans la zone nucléaire du Rubané, suggère que le fléchissement à plus de $120^{\circ}$ ait fait partie de la pratique originelle de cette culture. Ces hyperflexions devaient ainsi probablement impliquer le recours à des liens ou à une enveloppe enserrant les jambes des défunts. La mise en parallèle de ces éléments avec les données recueillies dans la plaine d'Alsace appuie donc une nouvelle fois le conservatisme des communautés de Haute-Alsace par rapport à la pratique initiale [51], tandis que celles de Basse-Alsace s'en affranchissent au moins à partir du Rubané récent en procédant au dépôt des membres inférieurs selon des degrés de flexion extrêmement variables.

[51] On notera que les hyperflexions des membres inférieurs sont également visibles dans le Bassin parisien qui, rappelons-le, fait partie du RSO.

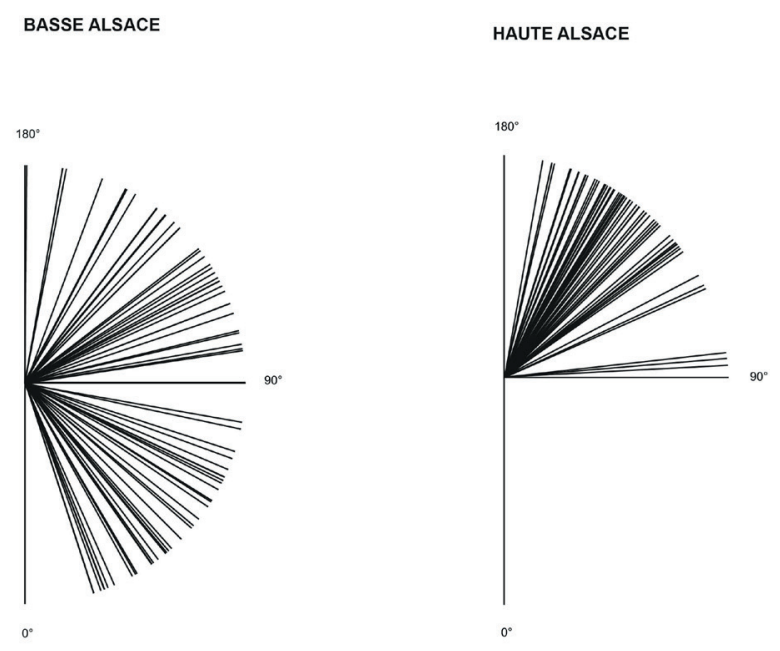

Fig. 8 : degrés de flexion des membres inférieurs des défunts de Basse et Haute-Alsace au Rubané (toutes étapes confondues).

DAO : Laura Waldvogel.
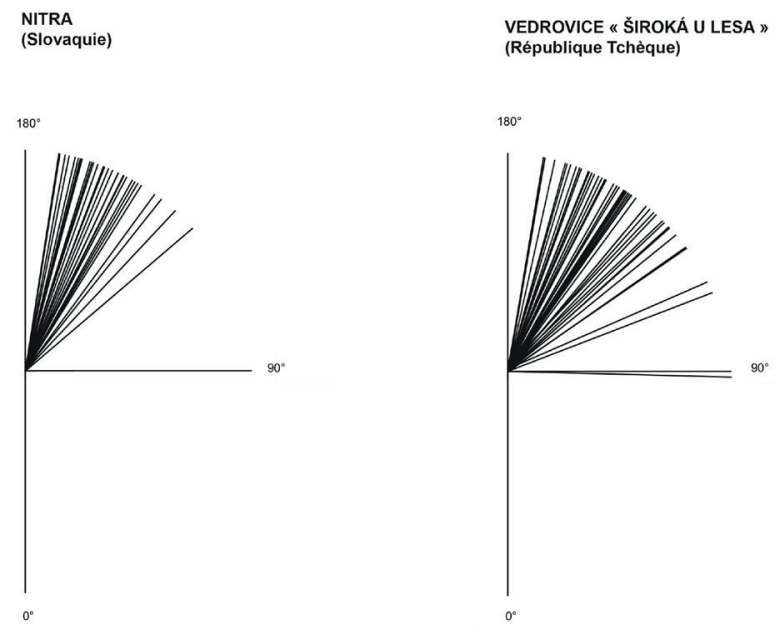

Fig. 9 : degrés de flexion des membres inférieurs des défunts des nécropoles de Nitra (Slovaquie) et de Vedrovice «Široká u lesa » (République Tchèque). DAO : Laura Waldvogel.

\section{SCHWETZINGEN}

(Allemagne)

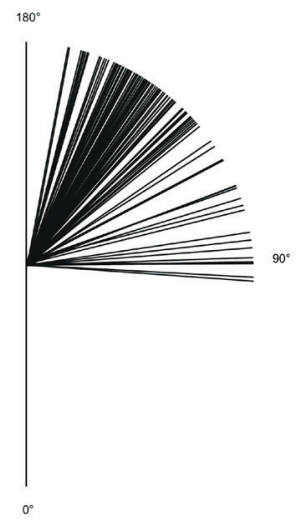

Fig. 10 : degrés de flexion des membres inférieurs des défunts de la nécropole de Schwetzingen (Allemagne). DAO : Laura Waldvogel. 


\section{LA BASSE-ALSACE : CAS À PART DE LA TRADITION II ?}

La poursuite de notre analyse a toutefois révélé que cette variabilité dans le degré de flexion des membres inférieurs n'est pas caractéristique de la tradition II, comme le montre le cas de Schwetzingen (Rubané récent). Sur les 104 défunts étudiés, 11 présentent des angles compris entre $85^{\circ}$ et $110^{\circ}$ - les 93 restants étant déposés selon des angles allant de $110^{\circ}$ à $169^{\circ}$ (fig. 10). Cette démarche, qui sera bientôt étendue à d'autres ensembles funéraires de la tradition II, accentue une nouvelle fois l'existence de spécificités en Basse-Alsace à partir de l'étape récente par rapport aux groupes du RN et du RNO. Celles-ci avaient en effet déjà été suggérées par la fréquence de la position allongée sur le dos, les membres inférieurs en extension, qui est beaucoup plus discrète hors de Basse-Alsace : elle est par exemple relevée dans 2 cas (sépultures 133 et 143) à Schwetzingen. Ces particularités sont probablement révélatrices de divisions internes régnant dès le Rubané récent en Basse-Alsace [52], en cela qu'elle touche l'un des cœurs de la pratique funéraire : la position. Sans nous attarder ici sur les origines possibles de ces nouveaux gestes, nous nous contenterons de signaler qu'ils trahissent une volonté partagée par certains individus de proposer un point de vue différent sur le passé, soit en réinterprétant des gestes originels (degré de flexion des membres inférieurs aléatoires), soit en marquant une rupture nette avec ces derniers (position allongée sur le dos, à laquelle s'ajoutent les orientations « antipodiques » caractéristiques de l'ensemble de la tradition II).

\section{INDICES DE VARIABILITÉS GÉOGRAPHIQUES DANS LES TRADITIONS I ET II À PARTIR DU RUBANÉ RÉCENT}

L'examen préliminaire d'une partie des sépultures de I'ouest du Rubané occidental suggère que les dissemblances entre le RBA d'une part, et le RNO et le RN d'autre part ne sont pas propres à la tradition II. Des spécificités semblent en effet se détacher au sein de la tradition I à partir du Rubané récent : elles concernent aussi bien la composition du mobilier funéraire que la structure de la fosse sépulcrale. Ces variabilités sont d'ordre géographique et expliquent en grande partie les différentes trajectoires relevées à partir du Rubané récent [53]. Tel est le cas de la récurrence, au Rubané récent et final du Bassin de la Seine [54], de niches latérales et de banquettes [55] dans les fosses sépulcrales du Bassin parisien - des structures que I'on pensait encore être anecdotiques il y a quelques années [56]. Ces aménagements sont en effet inconnus en Basse et Haute-Alsace malgré la qualité des fouilles conduites dans les deux régions [57], à l'exception de la tombe $2014 / 5$ de Mulhouse-Est, qui comporte une banquette dans sa partie est [58], mais pas de niche. Ces systèmes, dont le nombre est probablement sous-estimé [59], ont notamment été identifiés à Berry-au-Bac « Le Vieux Tordoir » [60] (5 tombes), à Écriennes « La Folie » (16 tombes) [61], à Bucy-le-Long « La Fosselle » [62] (5 tombes), à Menneville «Derrière le Village » (1 tombe) et à Maizysur-Aisne « Les Grands Aisements » (1 tombe) [63]. Un second exemple de cette variabilité géographique concerne le dépôt de céramiques auprès des défunts au cours du Rubané récent et final : alors que les vases constituent les objets les plus déposés dans le Bassin parisien, suivant la pratique de la zone nucléaire [64],

du Prépoux », datée du Villeneuve-Saint-Germain (VSG). Voir Prestreau 1992, p. 181 ; Thevenet 2004, p. 824. [56] BonnABel et al. 2003 ; Constantin et al. 2003 ; THEVENET 2004.

[57] JeUnesse 2003, p. 22.

[58] JeUneSSE et al. 2015, p. 30-37. La particularité de cette tombe réside également dans le traitement réservé au défunt, caractérisé par un probable prélèvement massif de ses restes après décomposition des chairs ainsi qu'un concassage des os restants.

[59] L'arasement trop important des tombes et la concavité latérale de la fosse ont probablement contribué à empêcher leur observation par le passé (BonNABEL et al. 2003, p. 53).

[60] AlLARD et al. 1997.

[61] BonNABEL et al. 2003.

[62] HACHEM et al. 1998.

[63] TheVenet 2004, p. 819-822.

[64] Voir notamment ALLARD et al. 1997, p. 35-36 pour la vallée de l'Aisne. 


\begin{tabular}{|c|c|c|c|c|}
\hline & \multicolumn{2}{|c|}{ TRADITION I } & \multicolumn{2}{|c|}{ TRADITION II } \\
\hline & $\begin{array}{c}\text { la } \\
\text { (Haute-Alsace, Hegau) }\end{array}$ & $\begin{array}{c}\mathbf{l b} \\
\text { (Bassin parisien) }\end{array}$ & $\begin{array}{c}\text { Ila } \\
\text { (Basse Alsace) }\end{array}$ & $\begin{array}{c}\text { Ilb } \\
\text { (groupes du RNO } \\
\text { et du RN) }\end{array}$ \\
\hline 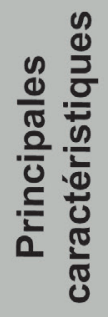 & $\begin{array}{l}\text { - Absence du } \\
\text { système de banquette } \\
\text { et de niche latérale ; } \\
\text { - Exclusion des } \\
\text { céramiques dans le } \\
\text { mobilier funéraire. }\end{array}$ & $\begin{array}{l}\text { - Fréquence du } \\
\text { système de banquette } \\
\text { et de niche latérale ; } \\
\text { - Présence de } \\
\text { céramiques dans le } \\
\text { mobilier funéraire. }\end{array}$ & $\begin{array}{l}\text { - Fréquence des individus } \\
\text { déposés sur le dos, membres } \\
\text { inférieurs en extension; } \\
\text { - Fréquence des individus } \\
\text { présentant des membres } \\
\text { inférieurs fléchis } \\
\text { aléatoirement. }\end{array}$ & $\begin{array}{l}\text { - Faible nombre d'individus } \\
\text { déposés sur le dos, membres } \\
\text { inférieurs en extension; } \\
\text { - Absence d'individus } \\
\text { présentant des membres } \\
\text { inférieurs fléchis à moins } \\
\text { de } 85^{\circ} \text {. }\end{array}$ \\
\hline 电 & $\begin{array}{l}\text { Ensisheim "Les Octrois », } \\
\text { Wittenheim "Le Moulin » }\end{array}$ & $\begin{array}{c}\text { Berry-au-Bac " Le } \\
\text { Vieux Tordoir », } \\
\text { Ecriennes " La Folie ", } \\
\text { Bucy-le-Long } \\
\text { "La Fosselle », } \\
\text { Maizy-sur-Aisne } \\
\text { "Les Grands Aisements" }\end{array}$ & $\begin{array}{l}\text { Vendenheim "Le Haut du } \\
\text { Coteau », Quatzenheim "Auf } \\
\text { dem Spitzenfad », } \\
\text { Souffelweyersheim } \\
\text { "Tuilerie Reiss und Bund », } \\
\text { Ingenheim "Bannenberg » }\end{array}$ & Schwetzingen \\
\hline
\end{tabular}

Fig. 11 : tentative de division des traditions I et II en sous-groupes au Rubané récent et final. DAO : Laura Waldvogel.

ils semblent être totalement exclus du mobilier funéraire en Haute-Alsace [65]. Ces premiers éléments, combinés aux résultats de l'étude de la « grammaire » des membres inférieurs des défunts, permet d'ores et déjà d'envisager l'existence de deux sous-groupes au sein de chacune des deux traditions funéraires, que l'on nommera tradition Ia et Ib, ainsi que tradition IIa et IIb (fig. 11). Notre tentative de subdivision a vocation à être parachevée dans le cadre de travaux ultérieurs qui permettront peut-être d'identifier de nouvelles particularités géographiques et/ou chronologiques. Aussi doit-elle, pour l'instant, être considérée comme une proposition heuristique.

\section{CONCLUSION}

L'étude de la « grammaire » des membres inférieurs des défunts, qui s'est concentrée dans un premier temps sur la plaine d'Alsace avant d'être étendue à d'autres sites rubanés, a mis en évidence un nouvel indice appuyant I'hypothèse d'une variabilité géographique visible dès le Rubané récent et relative à la position des individus de la tradition II. Des recherches préliminaires nous ont toutefois amené à constater que cette variabilité géographique n'est pas spécifique à cette tradition et qu'elle est également perceptible dès la même étape dans certaines zones de la tradition I (Haute-Alsace et Bassin parisien), où elle concerne a minima la structure de la fosse sépulcrale. Les deux traditions funéraires de l'ouest du Rubané occidental semblent, de fait, être plus complexes qu'initialement postulé et présenteraient une variabilité de plusieurs ordres dont les éléments peuvent être isolés au sein de « sous-entités » distinctes. Ainsi, la variabilité géographique que nous avons identifiée nous a conduit à distinguer ce que nous appellerons provisoirement les « sous-groupes » Ia et $b$, ainsi que IIa et $b$. Tandis que les changements relevés dans les «sous-groupes» Ia et b n'affectent pas le système caractéristique de la tradition I, ceux relevés dans le « sous-groupe » IIa touchent directement celui de la tradition II, sans pour autant marquer une rupture nette avec celui-ci. La nature intrinsèque des modifications ayant pris place dans les «sous-groupes » Ia/b et IIa semble ainsi indiquer une distanciation plus prononcée des communautés de tradition IIa par rapport à l'interprétation communément transmise du passé commun. Les changements connus par la tradition ne peuvent en effet, comme le souligne G. Lenclud, « s'opérer que sur fond de continuité » [66]. Ils sont alors révélateurs de l'émergence de nouvelles interprétations du passé liée à des enjeux contemporains, car il s'agit de « trouver dans [le passé] l'esquisse de solutions que nous croyons justes aujourd'hui non parce qu'elles ont été pensées hier mais parce que nous les pensons maintenant »[67].

[65] Voir les nécropoles d'Ensisheim « Les Octrois » et de Wittenheim « Le Moulin ».

[66] LENCLUD 1987, p. 113-116.

[67] PouilLon 1975, p. 60. 


\section{BASSE-ALSACE (TRADITION IIA)}

- Bischoffsheim «AFUA du stade » : sépulture 4000. Bibliographie : Lefranc et al. 2004.

- Entzheim « Sud-est de la gare » : sépulture II. Bibliographie : ForRER 1912.

- Entzheim «Terres de la Chapelle - ZAC » : sépultures 617, 962 et 2005. Bibliographie : LefrANC 2012.

- Ingenheim «Bannenberg » : sépultures 158, 277 et 280. Bibliographie : Lefranc et al. 2014.

- Oberschaeffolsheim «Zwischen Oberschaeffolsheim und Ittenheim » : sépulture 1. Bibliographie : ForRER 1916.

- Osthoffen-Breuschwickersheim : sépulture 2. Bibliographie : Forrer 1916.

- Quatzenheim «Auf dem Spitzenpfad » : sépultures 6 et 12. Bibliographie : Stieber 1947 ; StIeber 1955.

- Rosheim «ZAC du Rosenmeer - Extension Baruch » : st. 93. Bibliographie : Lefranc et al. 2014.

- Souffelweyersheim « Tuilerie Reiss und Bund » : sépultures Q, R, S, T, 30, 32, 38, 40, 41, 48, 49 et 50. Bibliographie : ULRICH 1939 ; ULRICH 1953.

- Stutzenheim « Über dem Stutzenweg » : point XI. Bibliographie : STIEbER 1952.

- Vendenheim «Le Haut du Coteau » : sépultures 8, 12, 15, 20, 86, 87, déf. 1 de la sépulture 91 , déf. 2 de la sépulture $93,98,104,105,112,124,130$, déf. 2 de la sépulture 133, 134, 138, 139, 140, 141, 148, 159, 160, 161, 167, 173, 177, 179, 184, 186, 193 et 194. Bibliographie : JEUNESSE 2002.

\section{HAUTE-ALSACE (TRADITION IA)}

- Brunstatt «Lang Dudely Acker» : sépulture 1. Bibliographie : THeVEnIN 1978.

- Ensisheim «Les Octrois » : sépultures 3, 4, 5, 6, 8, 12, 15, 16, 17, 19, 20, 21, 22, 23, 24, 26, 27, 29, 30, 31, 32, 34, 35, 37, 38, 39, 40, 44 et 45. Bibliographie : LAMBACH 1993 ; JeUneSSE 1995b ; JeUnesSe \& BoËs 1996.

- Ensisheim « Ratfeld » : sépulture 63. Bibliographie : JeUnesse \& SAINTY 1992.

- Geispitzen « Stuecke » : st. 1, 2, 6 et 7. Bibliographie : Wolff et al. 2000.

- Morschwiller-le-Bas « Ungeheuer Hoelzle » : sépultures 4, 5 et 54. Bibliographie : Vergnaud et al. 2016.

- Mulhouse-Est : sépultures 2, 3, 5, 6, 11, 12, 13, 14, déf. 1 de la sépulture 15, 16, 17, 18, 19, 20 et $2014 / 4$. Bibliographie : Schweitzer \& Schweitzer 1977 ; Jeunesse et al. 2015.

- Wettolsheim «Ricoh » : sépulture 359. Bibliographie : JEUNESSE 1988.

- Wittenheim «Le Moulin »: sépultures 330, 342, 359, défunts 1 et 2 de la sépulture 530/635. Bibliographie : GuiLLOTIN 2011.

\section{BIBLIOGRAPHIE}

Allard, Pierre et al., 1997, «Premiers éléments sur cinq tombes rubanées à Berry-au-Bac (Aisne, France) : principaux apports à l'étude du rituel funéraire danubien occidental », dans Christian Jeunesse (dir.), Actes du 22e Colloque Interrégional sur le Néolithique. Strasbourg 27-29 octobre 1995, Supplément aux Cahiers de l'Association pour la Promotion de la Recherche Archéologique en Alsace, Strasbourg, p. 31-43.

Bonnabel, Lola et al., 2003, «Un groupe de tombes en contexte d'habitat néolithique rubané à Écriennes «La Folie » (Marne) : approche des gestes funéraires », dans Philippe Chambon \& Jean Leclerc (dir.), Les pratiques funéraires néolithiques avant 3500 av. J.-C. en France et dans les régions limitrophes (Saint-Germain-en-Laye, 15-17 juin 2001), Mémoires de la Société Préhistorique Française 33, p. 45-53.

Bouju, Jacky, 1995, « Tradition et identité », Enquête 2, p. 95-117.

Boulestin, Bruno, 2016, «Norme funéraire : illusions et vérités», dans Michel Lauwers \& Aurélie Zemmour (dir.), Qu'est-ce qu'une sépulture ? Humanités et systèmes funéraires de la Préhistoire à nos jours, XXXVI rencontres internationales d'archéologie et d'histoire d'Antibes, Antibes, p. 363-377.

Constantrn, Claude et al., 2003, « Les tombes rubanées de la vallée de l'Aisne. Présentation », dans Philippe Chambon \& Jean Leclerc (dir.), Les pratiques funéraires néolithiques avant 3500 av. J. -C. en France et dans les régions limitrophes (Saint-Germain-en-Laye, 15-17 juin 2001), Mémoires de la Société Préhistorique Française 33, p. 56-63. 
Doнrn-Iнміg, Margarete, 1983, « Das bandkeramische Gräberfeld von Aldenhoven-Niedermerz, Kreis Düren », dans Gerhard Bauchhenss (dir.), Archäologie in den Rheinischen Lössbörden, Beiträge zur Siedlungsgeschichte im Rheinland, Köln, p. 47-190.

DudaY, Henri et al., 1990, «L'Anthropologie "de terrain" : reconnaissance et interprétation des gestes funéraires », Bulletins et Mémoires de la Société d'anthropologie de Paris 2/3-4, p. 29-49.

Duday, Henri \& Boulestin, Bruno, 2005, «Ethnologie et archéologie de la mort : de l'illusion des références à I'emploi d'un vocabulaire », dans Claude Mordant \& Germaine Depierre (dir.), Les pratiques funéraires à l'âge du Bronze en France, Sens-en-Bourgogne, p. 17-35.

FORRER, Robert, 1912, « Das Neolitische Gräberfeld bei Lingolsheim verglichen mit unseren bandkeramischen Gräbern 》, Anzeiger für Elsässische Altertumskunde 12, p. 215-231.

FORRER, Robert, 1916, «Spondylus-Muschelschmuck der Steinzeit aus dem Elsass », Anzeiger für Elsässische Altertumskunde 2, p. 715-726.

ForRer, Robert \& JÄNGer, Fernand, 1918, « Neolithisches Gräberfeld bei Hönheim-Suffelweyersheim », Anzeiger für Elsassische Altertumskunde 33-36, p. 875-886.

Gerling, Claudia, 2012, «Das Linearbandkeramische Gräberfeld von Schwetzingen, Rhein-Neckar-Kreis », Fundberichte aus Baden-Württemberg 32/1, p. 8-263.

DOI : 10.11588/ai.2007.1.11153.

Gurllotrn, Stéphanie, 2011, Wittenheim Lotissement «Le Moulin » (Alsace, Haut-Rhin (68)), Rapport final d'opération d'Archéologie préventive, SRA Alsace.

HAChem, Lamys et al., 1998, « Le site rubané de Bucy-le-Long “La Fosselle” (Aisne) », Internéo 2, p. 17-27.

IletT, Michael \& Meunier, Katia, 2013, « Avant-propos », Bulletin de la Société préhistorique française 110/3, p. 415-420.

Jeunesse, Christian, 1988, « Wettolsheim-Ricoh. Du Néolithique à l'ère postindustrielle. Un sauvetage archéologique dans la plaine d'Alsace », Catalogue d'exposition, Association pour la Recherche Archéologique en Alsace, Horbourg-Wihr. Jeunesse, Christian, 1995a, « Les groupes régionaux occidentaux du Rubané (Rhin et Bassin parisien) à travers les pratiques funéraires », Gallia préhistoire 37, p. 115-154.

Jeunesse, Christian, 1995b, Rapport de fouille programmée. Campagne de fouille 1995 sur la nécropole d'Ensisheim «Les Octrois » (Haut-Rhin), Rapport de fouille programmée, SRA Alsace.

JeUnesse, Christian, 1996, «Variabilité des pratiques funéraires et différenciation sociale dans le Néolithique ancien danubien », Gallia préhistoire 38, p. 249-286.

Jeunesse, Christian, 1997, Pratiques funéraires au Néolithique ancien. Sépultures et nécropoles danubiennes (55004900 av. J.-C.), Paris.

Jeunesse, Christian, 2002, Vendenheim « Le Haut du Coteau ». Une nécropole du Néolithique ancien, Document final de synthèse, SRA Alsace, 3 vol.

Jeunesse, Christian, 2003, «Les pratiques funéraires du Néolithique ancien danubien et l'identité rubanée : découvertes récentes, nouvelles tendances de la recherche », dans Philippe Chambon \& Jean Leclerc (dir.), Les pratiques funéraires néolithiques avant 3500 av. J.-C. en France et dans les régions limitrophes (Saint-Germain-en-Laye, 15-17 juin 2001), Mémoires de la Société Préhistorique Française 33, p. 19-32.

Jeunesse, Christian, 2005, « Nouvelles données sur la nécropole du Néolithique ancien de Quatzenheim (Bas-Rhin) 》, Cahiers Alsaciens d'Archéologie d'Art et d'Histoire XLVIII, p. 5-30.

Jeunesse, Christian, 2008, « Variations stylistiques et formation des groupes régionaux dans le Rubané occidental. L'exemple des décors orthogonaux », dans Frank Falkenstein, Sabine Schade-Lindig \& Andrea Zeeb-Lanz (dir.), Kumpf, Kalotte, Pfeilschaftglätter. Zwei Leben für die Archäologie. Gedenkschrift für Anne-marie Häusser und Helmut Spatz, Rahden, Marie Leidorf (Internationale Archäologie - Studia honoraria, 27), p. 129-151.

Jeunesse, Christian \& SaInty, Jean, 1992, «L'habitat néolithique ancien du site d'Ensisheim « Ratfeld » (Haut-Rhin). Structures, céramique rubanée, céramique non rubanée, parure », Cahiers de l'Association pour la Promotion de la Recherche Archéologique en Alsace 8, p. 95-145.

JeUnesse, Christian \& BoËs, Éric, 1996, Rapport de fouille programmée. Campagne de fouille 1996 sur la nécropole rubanée d'Ensisheim «Les Octrois » (Haut-Rhin), Rapport de fouille programmée, SRA Alsace.

JeUnesse, Christian et al., 2015, Illzach «Steinlache » (nécropole de Mulhouse-Est), Rapport de fouille, SRA Alsace. KaPANDJI, A. I., 2018, Anatomie fonctionnelle 2 : le membre inférieur, Paris (7e édition).

Lambach, François, 1993, «La nécropole rubanée d'Ensisheim "Les Octrois". Description des tombes et anthropologie de terrain », Cahiers de I'Association pour la Promotion de la Recherche Archéologique en Alsace 9, p. 17-48. 
LeCLeRc, Jean, 1990, «La notion de sépulture », Bulletins et Mémoires de la Société d'anthropologie de Paris 2/3-4, p. 13-18.

Lefranc, Philippe, 2012, Entzheim, Bas-Rhin, In der Kappell Z.A. « Les Terres de la Chapelle 2 ». Village rubané, enceinte et inhumations de la fin du Ve millénaire et habitat Michelsberg, Rapport de fouille, SRA Alsace, 3 vol.

Lefranc, Philippe, 2013, « Les relations entre les groupes rubanés d'Alsace et du bassin de la Seine : I'apport des styles céramiques », Bulletin de la Société préhistorique française 110/3, p. 495-512.

Lefranc, Philippe et al., 2004, « Bischoffsheim « AFUA du stade », DFS de fouilles de sauvetage urgent, SRA Alsace. Lefranc, Philippe et al., 2014, « La nécropole Rubané récent d'Ingenheim “Bannenberg" (Bas-Rhin) », dans Philippe Lefranc, Anthony Denaire \& Christian Jeunesse (éd.), Données récentes sur les pratiques funéraires néolithiques de la Plaine du Rhin supérieur, Oxford, p. 5-58.

LeNCLud, Gérard, 1987, «La tradition n'est plus ce qu'elle était... Sur la notion de "tradition" et de "société traditionnelle" en ethnologie », Terrain 9, p. 110-123.

Mauss, Marcel, 1950, Sociologie et anthropologie, Paris.

Modderman, Pieter Jan Remees, 1970, Linearbandkeramik aus Elsloo und Stein, Leiden.

Pavúk, Juraj, 1972, « Neolithisches Grâberfeld in Nitra », Slovenska Archeologia 20/1, p. 5-106.

Pourllon, Jean, 1975, « Tradition : transmission ou reconstruction », dans Jean Pouillon (dir.), Fétiches sans fétichisme, Paris, p. 155-173.

Pourllon, Jean, 1991, «Tradition », dans Pierre Bonté \& Michel Izard (éd.), Dictionnaire de l'Ethnologie et de l'Anthropologie, Paris, p. 710.

Prestreau, Michel, 1992, « Le site néolithique et protohistorique des Falaises de Prépoux à Villeneuve-la-Guyard (Yonne) », Gallia préhistoire 34, p. 171-207.

Schweitzer, Roger \& Schwertzer, Joël, 1977, « La nécropole du Danubien moyen de Mulhouse-Est », Bulletin du Musée Historique de Mulhouse 84, p. 11-63.

Streber, Arthur, 1947, «Le cimetière néolithique à céramique rubanée de Quatzenheim (Bas-Rhin) », Cahiers Alsaciens d'Histoire et d'Archéologie 128, p. 21-30.

Streber, Arthur, 1952, « Fouilles dans les stations néolithiques de Stutzheim (Bas-Rhin) », Cahiers Alsaciens d'Histoire et d'Archéologie CXXXII, p. 21-28.

Streber, Arthur, 1955, «Quatre nouvelles tombes du cimetière néolithique à céramique rubanée de Quatzenheim », dans Congrès Préhistorique de France, Strasbourg-Metz 1953, Paris, p. 594-606.

TABorin, Yvette, 1974, «La parure en coquillage de l'Épipaléolilhique au Bronze ancien en France », Gallia Préhistoire 17, p. 100-179.

Thevenet, Corinne, 2004, « Une relecture des pratiques funéraires du Rubané récent et final du Bassin parisien : I'exemple des fosses sépulcrales dans la vallée de l'Aisne », Bulletin de la Société préhistorique française 101/4, p. 815-826. DOI : $10.3406 /$ bspf.2004.13070

Thevenin, André, 1978, « Alsace », Gallia Préhistoire 21/2, p. 547-572.

TyLor, Edward Burnett, 1871, Primitive Culture, London.

UlRICH, Henri, 1939, « Le cimetière néolithique à céramique rubanée de Hœnheim-Souffelweyersheim », Cahiers Alsaciens d'Histoire et d'Archéologie 117-120, p. 9-24.

ULRICH, Henri, 1953, «Le cimetière néolithique à céramique rubanée de Hœnheim-Souffelweyersheim. Bilan d'ensemble avec les nouvelles sépultures », Cahiers d'Archéologie et d'Histoire d'Alsace 133, p. 21-36.

Vergnaud, Luc et al., 2016, Morschwiller-le-Bas « Ungeheuer Hoelzle ». Un ensemble funéraire rubané, un habitat Horgen et Bronze final, Rapport final d'opération d'archéologie préventive, SRA Alsace.

WolfF, Jean-Jacques et al., 2000, « Un nouvel ensemble funéraire rubané à Geispitzen "Stuecke" (Haut-Rhin) 》, Cahiers de I'Association pour la Promotion de la Recherche Archéologique en Alsace XVI, p. 37-49. 